\title{
Consumption of ultra-processed foods and the risk of overweight and obesity, and weight trajectories in the French cohort NutriNet-Santé
}

\author{
Bernard Srour $^{1,2}$, Marie Beslay ${ }^{1}$, Caroline Méjean ${ }^{3}$, Benjamin Allès ${ }^{1}$, Thibault Fiolet ${ }^{1}$, \\ Charlotte Debras ${ }^{1}$, Eloi Chazelas ${ }^{1}$, Mélanie Deschasaux ${ }^{1}$, Serge Hercberg ${ }^{1,4}$, Pilar Galan ${ }^{1}$, \\ Carlos A. Monteiro ${ }^{5}$, Emmanuelle Kesse-Guyot ${ }^{1}$, Mathilde Touvier ${ }^{1,6}$ and Chantal Julia ${ }^{1,6}$ \\ ${ }^{1}$ Nutritional Epidemiology Research Team (EREN) - Inserm U1153, INRA U1125, CNAM, University of Paris 13, \\ Bobigny, France, \\ ${ }^{2}$ equal contributions, first author, France, \\ ${ }^{3}$ UMR MOISA INRA, Montpellier, France, \\ ${ }^{4}$ Avicenne hospital - Public Health department, Bobigny, France, \\ ${ }^{5}$ University of Sao-Paulo, Sao-Paulo, Brazil and \\ ${ }^{6}$ equal contributions, last author, France
}

\begin{abstract}
Introduction: Previous epidemiological studies have found associations between the consumption of ultra-processed foods and the risk of obesity-related outcomes, such as post-menopausal breast cancer, cardiovascular diseases, hypertension and mortality. However, only one Spanish prospective study has explored the associations between the consumption of ultra-processed foods and the risk of overweight and obesity. The aim of this study is to investigate the associations between ultra-processed food consumption and the risk of overweight and obesity, as well as the associations between ultra-processed food consumption and weight trajectories, in middle-aged adults included in the French large scale NutriNet-Santé cohort.
\end{abstract}

Methods: Overall, 110260 participants aged at least 18 years from the French NutriNet-Santé cohort (2009-2019) were included. Dietary intakes were collected using repeated 24 hour dietary records, merged with a food composition database of 3300 different products. These were categorized according to their degree of processing by the NOVA classification. Associations between ultraprocessed food intake and risks of overweight and obesity were assessed using multivariable Cox proportional hazard models. Associations between ultra-processed food intake and weight trajectories were assessed using multivariable linear mixed models for repeated measures with random slope and intercept. Models were adjusted for known risk factors (sociodemographic, lifestyle, and nutritional factors).

Results: Ultra-processed food intake was associated with a higher risk of overweight ( $\mathrm{n}=7063$ incident cases; hazard ratio for an absolute increment of 10 in the percentage of ultra-processed foods in the diet $=1.11(95 \%$ confidence interval 1.08 to 1.14$)$; $\mathrm{P}<0.0001$, median follow-up: 4.1y, 260304 person-years) and obesity $(\mathrm{n}=3066$ incident cases; $\mathrm{HR}=1.09(95 \%$ confidence interval 1.05 to 1.13$)$; $\mathrm{P}<0.0001$, median follow-up: $8.0 \mathrm{y} 365344$ person-years). Higher consumers of ultra-processed foods (4th quartile) were more likely to present an increase in body mass index over time (change of BMI/time-unit in Q4 vs Q1 $=0.04, \mathrm{P}<0.0001$ ). These results remained statistically significant after adjustment for several markers of the nutritional quality of the diet (fruits and vegetables and sugary drinks consumption, intakes of saturated fatty acids, sodium, sugar, dietary fiber or Healthy/Western patterns derived by principal component analysis) and after a large range of sensitivity analyses.

Conclusion: In this large observational prospective study, higher consumption of ultra-processed foods in the diet was associated with a higher risk of overweight and obesity. Public health authorities in several countries recently started to recommend privileging unprocessed/minimally processed foods and limiting ultra-processed food consumption.

\section{Conflict of Interest}

There is no conflict of interest 